\title{
Influence of Mate-Copying Tendencies through Simulated Attraction on Perceived Physical Attractiveness of Opposite Sex in Women
}

\author{
Magdalena A. Hanipraja; Bernadeth Deminiz; Adeline Dinda C; Nurul Arbiyah* \\ Faculty of Psychology, Universitas Indonesia, Depok, Indonesia \\ *E-mail: nurul.arbiyah31@ui.ac.id
}

\begin{abstract}
This study is a replication of Dunn and Doria's (2010) research, which intends to see the effect of matecopying tendencies among young adult women in perceiving the level of physical attractiveness of the opposite sex. Mate-copying tendencies can emerge in various forms, one of which is through simulated attraction that is shown through a photograph of a man surrounded by several women who show interested gestures towards him. The dependent variable was measured using a single item that measured perceived physical attractiveness of the male model in Likert scale (1-10). A total of 73 undergraduate students from Universitas Indonesia (average age $=19.6, \mathrm{SD}=1.48$ ) participated in this study. The statistical analysis result using independent sample t-test showed a significant difference in perceived physical attractiveness score between the experimental group and control group $(t(71)=2.048, p=0.044)$. The females who were given a photograph of the male model posing with several female models had a higher perceived physical attractiveness score for the male $(\mathrm{M}=5.24, \mathrm{SD}=1.342)$ than those who were given a stand-alone male model photo $(\mathrm{M}=4.61, \mathrm{SD}=1.293)$. The implications of this study on interpersonal relationships may explain why sometimes men who are engaged with other women are viewed as more attractive.
\end{abstract}

Keywords: mate-copying; perceived physical attractiveness; physical attractiveness; simulated attraction

\section{Introduction}

Physical attractiveness has become a critical issue to be studied upon in social psychology due to its influence on interpersonal processes, especially in romantic relationships (Dunn \& Doria, 2010; Langlois, Kalakanis, Rubenstein, Larson, Hallam, \& Smoot, 2000). Physical attractiveness is how the physical appearance of a person has evaluated aesthetically. Physical attraction is a determining factor in a person's first impression towards others, especially toward the opposite sex (Miller, 2015). Physical appearance is one of the first things a person would notice during an encounter. If the person is regarded as attractive, usually the person will be associated with relatively more positive traits, and a less attractive person with more negative traits; in other words, a person's physical appearance does correspond with the evaluation for the person's intangible qualities (Dunn \& Doria, 2010; Langlois, Kalakanis, Rubenstein, Larson, Hallam, \& Smoot, 2000). A physically attractive person is often more likable and is labeled as a person with "the most-wanted personality" compared to a less attractive person (Sparacino \&
Hansell, 1979, in Talbot, 2012). Based on research done by Dion, Bersheid, and Walster (1972), a good-looking person is perceived as more sensitive, kind-hearted, sociable, charming, friendly, strong, balanced, and energetic in comparison to a less attractive person. In line with the research, Miller (2015) further stated that a good-looking person is often associated with a good personality, or what is often called "what beautiful is good", as depicted by popular media. A physically appealing person is perceived as higher in the extraversion, agreeableness, and conscientiousness level (Miller, 2015). This evaluation can be based on selfperception, a perception of others, or a combination of both (Talbot, 2012), where someone can match his perception of his own physical attractiveness towards others around him. Furthermore, a perception of physical attractiveness of others can be influenced by factors such as the social environment.

The social environment helps shape the perception of physical attractiveness. A study by Jonason (2009) showed that a person with minimum experience in romantic relationships or minimum 
exposure to physically attractive people will consider a physical feature as invaluable in terms of relationship. Alas, the rate of a person's social experience interacts with his value towards physical attractiveness, enhancing his sensitivity towards physical evaluation. Moreover, culture is also suspected to have a role in shaping the standard for evaluating physical attractiveness, where different cultures produce different preferences. Swami (2015) in his research elaborated how westernization and globalization shape similar preferences of facial and other physical features as a defining attractive point in different cultures all over the world. Some research demonstrates the existence of social transmission in the physical attractiveness perception process, where an individual would be rated as more physically appealing when appearing with another person of the opposite sex. This type of social transmission is known as mate-copying.

Mate-copying is a mechanism found in both nonhuman animals and humans alike. Studies in guppies and primates show that in selecting their potential mates, they use cues provided contextually to aid the selection process. Female guppies, for example, will show a higher probability of selecting a male guppy that is presented with other female guppies surrounding it or when surrounded in a location previously habituated by said male guppy and another female guppy (Dugatkin \& Godin, 1993). This is due to the association between the number of females that have mated with the male guppy to the rate of possible succession-the more females, the higher rate of successful offspring. In humans, mate-copying is basically a strategy used to assist a person in mate-selection processes-in selecting high-quality potential mates (Rodeheffer et al, 2016). As found in guppies, humans - females, in particular - 'copy' selections from another human to help select a male with a higher quality, as exhibited by other females. In fact, men who are currently or have been in a relationship are regarded as more attractive (Uller \& Jonasson, 2003). Furthermore, researchers have found that matecopying tendencies are more commonly found in women rather than men, and this is due to the tendency that women use implicit social cues to aid their selection, therefore paying more attention to intangible qualities and often making error in their evaluation more than men (Thornhill \& Gangestad, 1999).

Mate-copying tendencies can be manifested in various forms of behavior. Research by Vakirtzis and Roberts (2012) used marital status of the male model to evaluate how women rate the attractiveness of the male model and found that married men are more desirable than single men. Other methods used to provide evidence for matecopying tendencies include forming simulated attraction (Dunn \& Doria, 2010). The mate-copying tendency is tested by comparing scores of perceived physical attractiveness of an opposite sex when presented with a same-sex model and when presented alone through a picture. The accompanying models are then instructed to simulate attraction towards the opposite sex model by showing subtle gestures that indicate 'attraction', such as a slight tilt of head towards the model or an interesting face.

This research is a replication of the one conducted by Dunn and Doria (2010), which examined matecopying tendency through visually presented simulated attraction. As explained previously, the experiment will involve participants individually presented with a picture of an opposite-sex model. Participants will be divided into two groups, with one group being presented the picture of the model accompanied by other models that serve as the simulated attraction. Previous research tested both genders for mate-copying tendency and its finding indicated that this mechanism is found only in females. Therefore, this current research will only be conducted on females to further validate the results of the predecessor research. We hypothesize that there will be a difference in perceived physical attractiveness when participants are presented with simulated attraction and without.

In general, related studies investigating matecopying tendencies have been conducted in western countries, such as England and the United States (Milonoff, et, al, 2007; Waynforth, 2007; Dunn \& Doria, 2010). This study is conducted to enrich the lack of similar study in the eastern culture, particularly Indonesia. Furthermore, the rise of online dating as a means of mate-selecting platform requires a quicker and more limited time in selecting potential mates. The lack of face-to-face and in-depth information regarding the potential mate also requires the use of some strategies to effectively select the mate, including scrolling through pictures (Finkel, et al, 2012). This study may provide an insight that might be useful to be considered when reviewing the context of online dating. 


\section{Methods}

Sample. Participants in this study were 73 female undergraduate college students of Universitas Indonesia. College students were chosen as romantic life is a vital part of young adults' stage of life. Participants were adult, heterosexual females, all of whom were active college students. Obtaining samples was done by accidental sampling method through an online application, obtained through Google Form and the information was broadcasted via online messaging platform (LINE). Participants were recruited through Google Form by submitting their personal data after first reviewing the requirements needed for participating. The form was broadcasted through various social media platforms and through direct offers from researchers to join the study at the place that the study was conducted. The age range for participants was $18-23$ years old $(\mathrm{M}=19.6, \mathrm{SD}=1.48)$. Afterward, the 73 participants were divided randomly into two groups, with 37 participants in the experimental group and 36 participants in the control group.

Research Design. The current study is an experimental study with a randomized between subject post-test only research design.

Instrument and Measurement. Researchers used a measuring instrument with 1-10 Likert Scale to measure perceptions of physical attractiveness of the male model appearing in the photos, where Scale $1=$ very unattractive and Scale $10=$ very attractive.

The question used to measure the perceived physical attractiveness was: "in your opinion, how attractive is the male in the following photo?"

This instrument is identical to that used in the previous study done by Dunn and Doria (2010).

Procedure. Before the experiment was conducted, a preliminary study was done to test the instrument used in the study, which took the form of a survey of the male models' photos. The preliminary survey was given to 72 female students in Faculty of Psychology in University of Indonesia. The survey was given to a specific group in order to control possible effects of familiarity in the study that will be conducted, thus the participants of this survey were noted to not be allowed to participate in the study. The survey was done to control ceiling effect by measuring the physical attractiveness of five models in the photos used for the experiment. The preliminary study was conducted through online form (Google Form). The participants were asked to rate the attractiveness of each model on a scale of 110 . The result of the preliminary study was used to determine the models who would be used for variable testing. The models picked were those who received the closest to average scores of physical attractiveness (4-5).

After the researchers determined the photos qualified for the experiment based on the preliminary study $(M=4.57)$, a total of 5 photos were selected to be used for testing, with only 1 photo serving as the measurement instrument and the remaining 4 photos acting as distractors. The researchers obtained the samples online. Participant candidates were asked to answer a few questions about themselves (age, gender, faculty, and sexual orientation).

Following the procedure in the exemplary study, the models in the photos are required to wear white shirt and the photos are taken in the same background in order to control various extraneous variables that may have emerged from the difference of outfit the models may have worn. Although previously not researched or elaborated further, there may have been possible extraneous variables when the outfit and overall elements of the photos were not controlled.

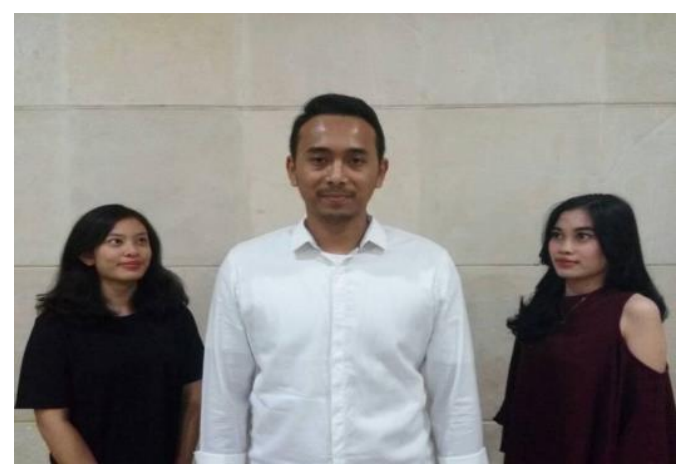

Figure 2.1. Experimental group

Table 2

Statistical testing result comparing perceived physical attractiveness score on experimental group and control group using t-test

\section{T-test result}

Figure 2.2. Controt group 
The study was done individually, in which each participant was seated in accordance with their respective group. Next, the researchers asked the participants to judge the physical attractiveness of the male model in the photo shown on a laptop screen, while simultaneously ignoring other characteristics that might be possessed by the model (e.g. kindness, clothes, etc.). The researchers showed 5 photos, with the first photo as the photo that was measured and the rest as distractors. The manipulation in this research was the appearance of female models accompanying every male model in the photos shown to the experimental group. Female models were positioned to stare at the male model while wearing the facial expression of someone "attracted" to the male model, to show stimulated attraction. The researchers did a verbal manipulation check on participants after the experiment was done. The questions asked for the manipulation check were, "Do you recognize the male model in the photos?" and "Do you realize the appearance of female models besides the male model?"

\section{Results}

Based on statistical analysis using independent sample t-test, a difference in score was found between the experimental group (shown photos with female models) and the control group (shown photos without female models).

Table 1.

Descriptive of perceived physical attractiveness of experimental group and control group

\begin{tabular}{lccc}
\hline Research groups & \multicolumn{3}{c}{ Change Score } \\
\cline { 2 - 4 } & $N$ & Mean & $S D$ \\
\hline Experimental group & 37 & 5.24 & 1.342 \\
Control group & 36 & 4.61 & 1.293 \\
Total & 73 & & \\
\hline
\end{tabular}

Note: $S D=$ Standard Deviation

The table above showed that the mean score for the experimental group was higher than the control group.

Based on the comparison of means between the two groups, a statistical analysis was done using independent sample t-test to determine if there was a significant difference in perceived physical attractiveness scores between the experimental and control groups. Indeed, the statistical analysis result showed a significant difference in perceived physical attractiveness score $(t(71)=2.048, \mathrm{p}<$ 0.05 , two-tailed) between the experimental group that was shown photos with female models $(M=$ $5.24, S D=1.342$ ) and the control group that was shown photos of a male model without female models $(M=4.62, S D=1.239)$, with an effect size of $r^{2}=0.056$. In other words, the independent variable, in this case, simulated attraction, has an effect of $r^{2}>0.056$ in affecting PPA, which, according to Gravetter and Walnau (2009) is classified as a small effect size. Other than that, this shows that $5.56 \%$ of the variance in perceived physical attractiveness can be explained by the presence of mate-copying tendencies in the form of simulated attraction.

\section{Table 2}

Statistical testing result comparing perceived physical attractiveness score on experimental group and control group using t-test

\begin{tabular}{llll} 
& \multicolumn{3}{c}{ T-test result } \\
\cline { 2 - 4 } & $T$ & $d f$ & sig. \\
\hline $\begin{array}{l}\text { Perceived } \\
\text { Physical }\end{array}$ & 2.048 & 71 & $0.044^{* *}$ \\
Attractiveness & & & \\
Score & & & \\
\hline
\end{tabular}

Note. ${ }^{*} p \leq .05 . * * p \leq .01 ; d f=$ degree of freedom; sig, $=$ significance

\section{Discussion \& Conclusion}

Based on statistical testing, a significant score difference in perceived physical attractiveness was found between the experimental group (with simulated attraction) and the control group (without simulated attraction). This result corresponds with prior research conducted by Dunn and Doria (2010). Physical attractiveness perception is one of the basic components in mate selection - therefore, a rating of physical attractiveness can be explained through potential mate selection mechanism, especially one oriented for a short-term relationship, as in shortterm relationships, physical attractiveness is a determining factor in mate selection (Thornhill and Gangestad, 1999). Below, we discuss arguments that support our finding.

First, mate-copying mechanism reduces sampling cost (Pomiankowski 1987, Reynolds \& Gross 1990, 
Andersson 1994, Dugatkin \& Höglund 1995 in Vakirtzis, 2011). According to Stohr (1998), when participants are given limited time and information in choosing their potential mate, they tend to use heuristic mechanisms to assist them in judging the quality of their potential mate. One of the heuristics used is judging based on face value, that is, physical attractiveness of the figure. The age range of the participants that is considered young (18-23) also contributes to the use of sampling cost-reducing mechanisms, where it is assumed that a relatively young person will have comparably fewer experience in mate selection, therefore using cues to assist selection to reduce sampling cost (Stöhr, 1998).

Perception of physical attractiveness also involves evaluating in an ambiguous situation, where there are factors contributing to the evaluation of attractiveness aside from physical features, such as social influences. Social influence in rating the physical attractiveness of the opposite sex is more commonly found in women, where the presence of other women acts as a social proof to validate the quality of the male model. Social proof is a form of informative influence, where the opinion of other people acts as a benchmark in an ambiguous situation (Deutsch \& Gerard, 1955). In other words, the presence of the female models around the male model while simulating attraction towards the male model acted as a social proof for the female participant, indicating that the male model has plausible qualities, hence the 'attraction' shown by the female models in the picture. This later influenced the evaluation of physical attractiveness, enhancing the score for the male model. Social proof as a validating tool for the male model quality can also be explained as a discriminative variable for the male quality, whether the male is a lowquality partner or a high-quality partner. The male model that received simulated attraction is regarded as a high-quality partner as proven by the female models around him (Townsend \& Levy, 1990; repercussions: Bateman, 1948; Trivers, 1972 in Rodeheffer et.al, 2016).

In regard to cross-cultural concerns corresponding with previous research, our result indicates a similar mechanism found in mate-copying in both Western and Eastern cultures. This implication is in line with research done by Li, Valentine, and Patel (2011), where a cross-cultural study examining sexual preferences in the United States and Singapore found that both countries have similar values in potential mate selection, especially in a short-term relationship context. The women in both countries are more dependent on social factors as a cue in mate selection rather than on direct physical features. The difference in the underlying qualities used to assess potential mates is more significantly found in a long-term relationship context and is not significantly influential in short-term relationships. But overall, universally, mate-copying mechanisms are similar throughout cultures all over the world.

In short, we conclude that there is an influence of mate-copying mechanism through simulated attraction on the perception of physical attractiveness of opposite sex among females. Even so, there are myriads of factors that underlie the process of mate-selection other than simulated attraction, such as individual preferences, intelligence, financial factors, etc.

There are some limitations to this research that may be noted. Theoretically, there is a possibility that the attractiveness of the accompanying female models may influence the evaluation; therefore, we suggest that future studies test and control said variable statistically. The age group in which the research was done within this study was arguably limited, thus there is a huge opportunity for further studies of the similar topic to be conducted in a wider agerange. Additionally, the experiment was done in Jakarta, which is a metropolitan city with an arguably global culture that may also be unrepresentative of Eastern Culture, especially indigenous culture- thus, it- is also suggested to conduct further cross-cultural studies to more thoroughly test this mechanism in other cultures. Moreover, the experiment was done by computer, thus limiting the time the participant had to view the male model which therefore meant that the environment was required to induce maximum attention. However, the experiment was conducted in a relatively open space, and although it was not especially bustling with a crowd, it may have been better were it conducted in a more secluded, closed space.

The result of this experiment implicates that young adult women in Indonesia tend to experience an enhanced perception of male attractiveness if said male is accompanied by other females simulated attraction through their gestures, indicating that the male has good qualities that attract the women.

This area of research is still vastly unexplored, and thus we deeply encourage future research to expand the knowledge on this topic. There are some 
possible variables that may affect the mechanism and influence mate-copying tendency in women, especially when used to aid the selection of a potential long-term partner. As stated by Scheib (2005, in Dunn \& Doria, 2010), there is a possible difference in evaluation in terms of selecting shortterm and long-term potential partners. Presenting information about general, intangible traits may also affect the rating of attractiveness. As stated before in the limitation of the study, we encourage future study to statistically control the rate of attractiveness of the accompanying female models, as it may have a role in the attractiveness rating of the male model. Dunn and Doria (2010) previously considered using video as an instrument, and this may be further discussed as it is possible to obtain different results when audio is included.

From the statistical analysis and previous discussion, it can be concluded that mate-copying tendency is found in young adult women in Indonesia, particularly undergraduate female students of Universitas Indonesia.

\section{Acknowledgements}

This article is an assignment done to fulfill the requirements to pass Research Methods and Inferential Statistics 2 class. The original experiment was done alongside with Nabila Aditya Perdana and Gladhys Eliora. The authors would also like to thank the models who posed for the photographs used for the instrument: Nugrah Maulid, Faisal Akbar, Gagah, Bagus, Ilham, Annisa, Alika, Dian, Kharisma, Marisa, and Ayu. The authors also thank Dian in her aid in proofreading the translation for this paper.

\section{References}

Deutsch, M., \& Gerard, H. B. (1955). A study of normative and informational social influences upon individual judgment. The Journal of Abnormal and Social Psychology, 51(3), 629

Dion, K., Berscheid, E., \& Walster, E. (1972). What is beautiful is good. Journal of Personality and Social Psychology, 24(3), 285.

Dugatkin, L. A., \& Godin, J. G. J. (1993). Female mate copying in the guppy (Poecilia reticulata): agedependent effects. Behavioral Ecology, 4(4), 289292

Dunn, M. J., \& Doria, M. V. (2010). Simulated attraction increases opposite sex attractiveness ratings in females but not males. Journal of Social, Evolutionary, and Cultural Psychology, 4(1), 1.
Finkel, E. J., Eastwick, P. W., Karney, B. R., Reis, H. T., \& Sprecher, S. (2012). Online dating: A critical analysis from the perspective of psychological science. Psychological Science in the Public Interest, 13(1), 3-66.

Geiselman, R. E., Haight, N. A., Kimata, L. G. (1984). Context Effects on the Perceived Physical Attractiveness of Faces, Journal of Experimental Social Psychology 20, 409-424

Gravetter, F. J., \& Wallnau, L. B. (2009). Statistics For The Behavioral Sciences (9th ed.) Belmont, C.A: Wadsworth.

Jonason, P. K. (2009). The value of physical attractiveness in partners: modeling biological and social variables. The Journal of Social Psychology, 149(2), 229-240.

Langlois, J. H., Kalakanis, L., Rubenstein, A. J., Larson, A., Hallam, M., \& Smoot, M. (2000). Maxims or myths of beauty? A meta-analytic and theoretical view. Psychological Bulletin, 126(3), 390-423.

Li, N. P., Valentine, K. A., \& Patel, L. (2011). Mate preferences in the US and Singapore: A crosscultural test of the mate preference priority model. Personality and Individual Differences, 50(2), 291294.

Miller, R. H. (2015) Intimate Relationships. McGrawHill: New York.

Milonoff, M., Nummi, P., Nummi, O., \& Pienmunne, E. (2007, January). Male friends, not female company, make a man more attractive. In Annales Zoologici Fennici (pp. 348-354). Finnish Zoological and Botanical Publishing Board.

Rodeheffer, C. D., Proffitt Leyva, R. P., \& Hill, S. E. (2016). Attractive Female Romantic Partners Provide a Proxy for Unobservable Male Qualities: The When and Why Behind Human Female Mate Choice Copying. Evolutionary Psychology, 14(2), 1474704916652144. Retrieved from http://journals.sagepub.com/doi/abs/10.1177/14747 04916652144

Stöhr. (1998). Evolution of mate-choice copying: a dynamic model. Animal Behaviour, 55(4), 893-903.

Swami, V. (2015). CulturalStöhr influences on body size ideals: unpacking the impact of westernization and modernization. European Psychologist, 20(1), 4451 .

Talbot, T. L. (2012). Comparing self-perception of attractiveness and overall life satisfaction: The differences between men and women (Order No. 1508917). Available from ProQuest Dissertations \& Theses Global. (1012103944). Retrieved from https://search.proquest.com/docview/1012103944?a ccountid $=17242$ 
Thornhill, R., \& Gangestad, S. W. (1999). Facial attractiveness. Trends in cognitive sciences, 3(12), $452-460$.

Uller, T. \& Johansson, L. C. (2003). Human mate choice and wedding ring effect: are married men more attractive?. Human Nature: An Interdisciplinary Biosocial Perspective, 14(3), 267-276.
Vakirtzis, A., \& Roberts, S. C. (2012). Human nonindependent mate choice: is model female attractiveness everything? Evolutionary Psychology, 10(2), 147470491201000205.

Waynforth, D. (2007). Mate choice copying in humans. Human Nature, 18(3), 264-271. 